Gideon Amir · Omer Angel · Bálint Virág

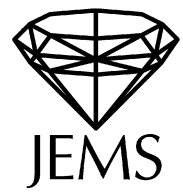

\title{
Amenability of linear-activity automaton groups
}

Received January 7, 2011 and in revised form April 11, 2011

Abstract. We prove that every linear-activity automaton group is amenable. The proof is based on showing that a random walk on a specially constructed degree 1 automaton group-the mother group-has asymptotic entropy 0. Our result answers an open question by Nekrashevych in the Kourovka notebook, and gives a partial answer to a question of Sidki.

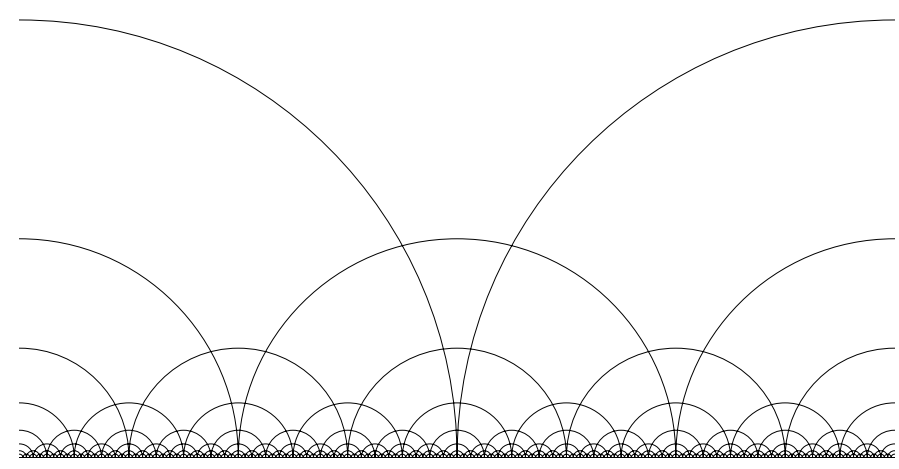

Fig. 1. A Schreier graph for a linear-activity group.

\section{Introduction}

Automaton groups are the core of the algebraic theory of fractals. Just as fractals do in geometry, automata groups form a rich new world within group theory. This world has been a source of many interesting examples, but until recently it resisted a general theory. Yet the simplicity of the definitions and the richness of examples suggest the existence of

G. Amir: Department of Mathematics, Bar-Ilan University, Ramat Gan, 52900, Israel; e-mail: gidi.amir@gmail.com

O. Angel: Department of Mathematics, University of British Columbia,

Vancouver, BC, V6T 1Z2, Canada; e-mail: angel@math.ubc.ca

B. Virág: Department of Mathematics, University of Toronto,

40 St George St., Toronto, ON, M5S 2E4, Canada; e-mail: balint@math.utoronto.ca 
such a theory. The goal of this paper is to make a step in this direction by proving that all linear-activity groups are amenable.

Automaton groups arise in various areas of mathematics; examples include:

The Grigorchuk group (see Grigorchuk, 1984), which has faster than polynomial but subexponential growth, answering the question of Milnor (1968) about the existence of such groups.

The Basilica group, a finitely generated amenable group containing a non-cyclic free semigroup. Its level Schreier graphs have a limit which is homeomorphic to the Basilica fractal, that is, the Julia set of the polynomial $z^{2}-1$, see Grigorchuk and Żuk (2002), Bartholdi, Grigorchuk, and Nekrashevych (2003, Figure 17 and Theorem 9.7), and Bartholdi and Virág (2005).

The Hanoi towers group, the group of possible moves in the Hanoi towers game on three pegs, a game introduced by Édouard Lucas in 1883. Its level Schreier graphs are discrete Sierpiński gaskets (see Grigorchuk and Šunić, 2006).

The long-range group, an interesting group whose Schreier graphs (Figure 1) were studied by Benjamini and Hoffman (2005) in the context of long-range percolation theory.

The lamplighter group on $\mathbb{Z}$, the first example of an infinite group with a Cayley graph that has discrete spectrum, providing also a counterexample to the strong Atiyah conjecture. See Grigorchuk and Żuk (2001).

Automaton groups are subgroups of the automorphism group Aut $\left(\mathbb{T}_{m}\right)$ of the rooted infinite $m$-ary tree for some $m$. Every element of Aut $\left(\mathbb{T}_{m}\right)$ can be decomposed as

$$
g=\left\langle\left\langle g_{0}, \ldots, g_{m-1}\right\rangle\right\rangle, \quad g_{i} \in \operatorname{Aut}\left(\mathbb{T}_{m}\right), \sigma \in \operatorname{Sym}(m),
$$

where the $g_{i}$, called first-level sections, now act on the subtrees rooted at the children of the root of $\mathbb{T}_{m}$, and $\sigma$ permutes these subtrees. An automaton $A$ is a finite subset of $\operatorname{Aut}\left(\mathbb{T}_{m}\right)$ so that first level sections of elements of $A$ are also in A. An automaton group is a group generated by an automaton. See, e.g., Sunic and Grigorchuk (2007) and references there for a general survey on automaton groups.

A systematic study of automaton groups was initiated by Sidki (2000), who introduced the concept of activity growth (or more briefly, activity), a measure of complexity for automaton groups. We defer the precise definition for later, but note that the activity can be either polynomial (of any degree) or exponential. There are exponential activity growth automaton groups that are isomorphic to the free group (see Glasner and Mozes, 2005; Vorobets and Vorobets, 2007). However, one expects polynomial activity automaton groups to be smaller. In particular, in contrast with most examples of finitely generated non-amenable groups, Sidki (2000) showed that polynomial activity automaton groups have no free subgroups (the works of Ol'shanski (1980), Adian (1982), and Olshanski and Sapir (2002), showed such groups exist (finitely generated, non-abelian with no free subgroup), but the examples were quite hard to come by). This prompted Sidki (2004) to ask the following natural question.

Question. Are all polynomial activity automaton groups amenable? 
It seems that the structure of polynomial automaton groups depends a lot on the degree, and we do not have a conjectured answer to Sidki's question. The first result in this direction is due to Bartholdi, Kaimanovich, and Nekrashevych (2008), who showed that all degree 0 (also called bounded) activity automaton groups are amenable. Their proof uses a variant of the self-similar random walk idea introduced in Bartholdi and Virág (2005) to prove the amenability of the Basilica group and later streamlined and generalized in Kaimanovich (2005). The goal of this paper is to show the following.

Theorem 1. All linear-activity automaton groups are amenable.

Example. A simple example of our theorem is given by the long-range group: Let $b$ act on integers by increasing them by 1 , and let $a$ act on integers by increasing them by the lowest power of 2 by which they are not divisible (with $a(0)=0$ ). The group generated by $a, b$ is called the long-range group; its Schreier graph is shown in Figure 1. Its automaton has two states defined recursively by

$$
a=\langle\langle a, b\rangle\rangle, \quad b=\langle\langle b, 1\rangle\rangle(01) .
$$

The question of the amenability of the long-range group was posed by Nekrashevych, in the Kourovka notebook (2006, Question 16.74), and also in Guido's book of conjectures (2008, Conjecture 35.9).

Our paper builds on previous work in the theory, including Bartholdi and Virág (2005), Kaimanovich (2005), and Bartholdi et al. (2008). The first part of the proof is to construct a family of linear-activity automaton groups - the mother groups-which are then shown to contain subgroups isomorphic to every linear-activity automaton group. This has been done in Bartholdi et al. (2008) for bounded automaton groups. We show how to perform this step for polynomial automata of any degree. It then suffices to show that the linearactivity mother groups are amenable.

The second part is to find a random walk on the mother group with zero asymptotic entropy. We can only do this for linear-activity mother groups. In fact, we conjecture that there is a phase transition for this question, namely such walks only exist up to degree 2 . Recall that a random walk is symmetric if its step distribution $\mu$ satisfies $\mu(g)=\mu\left(g^{-1}\right)$ for all group elements $g$.

Conjecture 2. The mother group of degree d has a symmetric random walk whose step distribution is supported on a finite generating set and whose asymptotic entropy is zero if and only if $d=0,1,2$.

For $d=1$ this follows from this paper. The high degree $(d \geq 3)$ case is proved $^{1}$ in Amir and Virág (2011). For $d=2$ this conjecture is still open.

Compared to previous work the random walks we consider are no longer self-similar, in the following sense.

The proof involves the analysis of random walks on an automaton group, by considering the action of the group elements on certain subtrees. This gives rise to a random

\footnotetext{
1 The conjecture is actually proved for all mother groups of degree 3 or more other than the degree 3 mother group over the binary alphabet. We believe this to be an artifact of the proof.
} 
walk on a subgroup that is isomorphic to the automaton group. A key difference between this and previous work is that the walks we consider are no longer self-similar, in that the projected random walk has a different support. In fact, while the initial distributions we consider have finite support, our proof involves the analysis of random walks with infinite support that arise through the evolution of the random walk step measure. The role previously played by self-similar walks is played by the evolution of random walk step measures that we can control. Such control requires a high level of regularity for the random walk. However, we believe that this is an artifact of the proof, and that the entropy bounds should hold for more general random walks.

Conjecture 3. Every symmetric random walk with boundedly supported step distribution on a polynomial automaton group with degree $d \leq 2$ has zero asymptotic entropy.

This conjecture is open even for $d=0$.

The main tool for the analysis of asymptotic entropy is study of the so-called ascension operator. Consider a random walk $X_{n}$ with step distribution $\mu$ on an automaton group, and let $v$ be the first child of the root of $\mathbb{T}_{m}$. The section of $X_{n}$ at $v$, looked at the times at which the walk fixes $v$ forms another random walk with step distribution $\mu^{\prime}$. The map $T: \mu \mapsto \mu^{\prime}$ is called the ascension operator (see further details in Section 4).

Ascension of random walks was first considered in Bartholdi and Virág (2005). The ascension operator in this form appeared in Kaimanovich (2005), where the asymptotic entropy inequality $H_{\infty}(\mu) \leq H_{\infty}(T \mu)$ was also proved. Iterating this inequality, one gets $H_{\infty}(\mu) \leq H_{\infty}\left(T^{n} \mu\right)$. In this paper, we will analyze $T^{n} \mu$ in the case when these measures are not finitely supported and not computable exactly. The following proposition allows us to relate $H_{\infty}(\mu)$ to the asymptotic entropy of limit points of the sequence of measures $T^{n} \mu$.

Proposition 4 (Upper semicontinuity of asymptotic entropy). If $v_{n}, v$ are probability measures on a countable group so that $v_{n} \rightarrow v$ weakly and $H\left(v_{n}\right) \rightarrow H(v)$, then

$$
\limsup _{n \rightarrow \infty} H_{\infty}\left(v_{n}\right) \leq H_{\infty}(v) .
$$

In light of Proposition 4, it suffices to find a subsequence along which $T^{n} \mu \rightarrow v$ and $H\left(T^{n} \mu\right) \rightarrow H(v)$, and $v$ has zero asymptotic entropy. Perhaps surprisingly, it is not too difficult to show that for appropriate $\mu$, any subsequential limit point $v$ has zero asymptotic entropy. For the other two claims, it suffices to show the tightness and entropytightness (defined below) of the sequence $T^{n} \mu$. Proving these facts takes up a large part of this paper. Showing the tightness of the sequence $T^{n} \mu$ is the main obstacle to extending our proof to the degree 2 case. For convenient reference, we summarize the preceding discussion in a theorem.

Theorem 5 (Asymptotic entropy of automaton groups). If the group generated by the support of $\mu$ acts transitively on all levels of the tree and the sequence $T^{k} \mu$ is entropytight, then for every subsequential weak limit point $v$ we have

$$
H_{\infty}(\mu) \leq H_{\infty}(v)
$$


The main challenge is to construct measures for which the ascension operator is tractable. The measures that we consider are based on the uniform measures $\overline{q_{i}}$ on certain finite subgroups of the mother group $\mathfrak{M}$. They have the property that if a probability measure $\mu$ on $\mathfrak{M}$ is a convex combination of convolution products of the $\overline{q_{i}}$, s, then so is $T \mu$ (i.e. the algebra generated by the $\overline{q_{i}}$ is invariant under $T$ ). Thus the iterated ascensions $T^{n} \mu$ of such measures can be understood in terms of the $\overline{q_{i}}$, s, which have extra symmetry and can be controlled. Further details can be found in Section 6.

Organization. The structure of this paper is as follows. Sections $2-4$ contain definitions, setup, background review and proof of some preliminary results; readers familiar with previous work on the subject may want to skip these sections. Section 2 reviews basic concepts related to automata groups. In Section 3 we do the same for entropy, and prove Proposition 4. In Section 4 we introduce and study the ascension operator. Mother groups are defined in Section 5, and it is shown that they contain all polynomial automata groups. In Section 6 we introduce a special class of measures, called patterns, and an algebraic way to study them. We also define an ascension operator for patterns. In Section 7 we study properties of iterated ascension on patterns. Sections 8 contains some preliminary results on entropy of pattern measures, and finally, the main theorem is proved in Section 9.

\section{Automata and their groups}

Basic definitions. Finite automata are the simplest interesting model of computing; we first connect our definition of automata to the more traditional one.

The space of words in alphabet $\{0, \ldots, m-1\}$ has a natural tree structure, with $\{w x\}_{x<m}$ being the children of the finite word $w$, and the empty word $\emptyset$ being the root. Let $\mathbb{T}_{m}$ denote this tree. A finite automaton on $m$ symbols is a finite set $A$ of states together with a map $A \rightarrow A^{m} \times \operatorname{Sym}(m)$ sending $a \mapsto\left(a_{0}, \ldots, a_{m-1}, \sigma_{a}\right)$. We will use the notation

$$
a=\left\langle\left\langle a_{0}, \ldots, a_{m-1}\right\rangle\right\rangle \sigma_{a} .
$$

An automaton acts on words in alphabet $\{0, \ldots, m-1\}$ sequentially. When the automaton is in a state $a$ and reads a letter $x$, it outputs $x . \sigma_{a}$ and moves to state $a_{x}$. From this state the automaton acts on the rest of the word. Symbolically, for a word $x w$ (starting with a letter $x$ ) we have the recursive definition

$$
(x w) \cdot a=\left(x \cdot \sigma_{a}\right)\left(w \cdot a_{x}\right) .
$$

The first $k$ symbols of the output are determined by the first $k$ symbols read, and the action is invertible. Note that the action is defined for both finite and infinite words, and the action on infinite words determines the action on finite words and vice versa. It follows that each element $a \in A$ is an automorphism of $\mathbb{T}_{m}$. The automaton group corresponding to an automaton $A$ is the subgroup of $\operatorname{Aut}\left(\mathbb{T}_{m}\right)$ generated by $A$. 
The action (1) corresponds to the following multiplication rule:

$$
\left.\left\langle a_{0}, \ldots, a_{d}\right\rangle \sigma \sigma\left\langle b_{0}, \ldots, b_{d}\right\rangle\right\rangle \tau=\left\langle\left\langle a_{0} b_{0 . \sigma}, \ldots, a_{m-1} b_{(m-1) . \sigma}\right\rangle\right\rangle \sigma \tau .
$$

This multiplication rule can be used to define automaton groups without any reference to automorphisms of the tree. However, keeping the action on the tree in mind makes some constructions used in the proof more natural.

We use the conjugation notation $a^{b}=b^{-1} a b$.

The notion of first-level sections can be generalized to any level. If $v \in \mathbb{T}_{m}$ is a finite word and $g \in \operatorname{Aut}\left(\mathbb{T}_{m}\right)$, then there is a word $v^{\prime}$ of equal length to $v$ and an automorphism $g^{\prime} \in \operatorname{Aut}\left(\mathbb{T}_{m}\right)$ such that $v w \cdot g=v^{\prime}\left(w \cdot g^{\prime}\right)$ for every word $w$. This $g^{\prime}$ is called the section of $g$ at $v$. Informally, $g^{\prime}$ is the action of $g$ on the subtree above the vertex $v$. The section of $g$ at $v$ is denoted $g(v)$.

Activity growth of automaton groups. For any state $a \in A$, the number of length- $n$ words $v$ such that the section $a(v)$ is not the identity satisfies a linear recursion. Thus this number grows either polynomially with some degree $d$ or exponentially. We define the degree of activity growth (for short, degree) of $a$ to be $d$ or $\infty$, respectively. The degree of an automaton group is the maximal degree of any of its generators. Automaton groups are said to have bounded, linear, polynomial or exponential activity growth when their degree is 0,1 , finite or infinite, respectively.

Degree and cycle structure. An automaton gives rise to a directed graph where there is a directed edge from a state $a$ to each of its first-level sections $a(i)$. If the same state appears more than once as an $a_{i}$ then there are parallel edges. If $a$ appears as a first-level section of itself (i.e., $a(i)=a$ ) then there is a loop at $a$. Thus the only edges leaving the identity (if it is a state of $A$ ) are loops back to the identity. The loops at the identity are called trivial cycles. To avoid degeneracies in the graph, we will assume from now on that all nonidentity states in the automaton act non-trivially. This assumption, which does not limit the generality of the automaton groups considered, allows some connections between the structure of the above graph and properties of the automaton.

The number of active vertices of $a$ at level $n$ is just the number of directed paths of length $n$ starting at $a$ and ending anywhere but the identity. It follows easily that an automaton is exponential if and only if there are two non-trivial cycles so that each is reachable from the other via a directed path.

The non-trivial directed cycles in the directed graph of a polynomial automaton have a partial order: $c_{1}<c_{2}$ if $c_{1} \neq c_{2}$ and there is a directed path from a state of $c_{2}$ to a state of $c_{1}$. Define the degree of a cycle $c$ as the maximal $n$ so that there is an increasing chain $c_{0}<c_{1}<\cdots<c_{n}=c$. It is straightforward to see that the degree of a polynomial automaton is the maximal degree of any cycle in its directed graph. The degree of a state $g$ is the maximal degree of a cycle reachable via a directed path from $g$. The identity 1 is always considered to have degree -1 . An automaton generates a finite group if and only if it contains no non-trivial cycle. 
Hierarchy levels. For a polynomial automaton, the hierarchy level of a state is a refinement of its degree, taking values in the sequence

$$
-\tilde{1},-1, \tilde{0}, 0, \tilde{1}, 1, \tilde{2}, 2, \ldots
$$

States in cycles of degree $d$ have hierarchy level $\tilde{d}$. States of degree $d$ that are not in a cycle have hierarchy level $d$. A state can point to states either in its own hierarchy level or in lower levels. The only state with hierarchy level $-\tilde{1}$ is the identity.

Collapsing levels. By dividing words in $m$ symbols into blocks of length $k$, we can view them as words in $m^{k}$ symbols. Similarly, given an automaton $A$ acting on $m$ symbols, it naturally gives rise to an automaton with the same states acting on words in $m^{k}$ symbols; we call this new automaton the $k$-collapse of $A$, and it acts on the tree $\mathbb{T}_{m^{k}}$.

Claim 2.1. The automaton groups of $A$ and of its $k$-collapse are isomorphic.

Proof. The key is that the vertices of $\mathbb{T}_{m^{k}}$ are naturally associated with every $k$ th level of $\mathbb{T}_{m}$, and that Aut $\left(\mathbb{T}_{m}\right) \subset \operatorname{Aut}\left(\mathbb{T}_{m^{k}}\right)$. Restricting this embedding to $G=\langle A\rangle$ gives an isomorphism from the automaton group of $A$ to the group of the $k$-collapse of $A$.

\section{Entropy and asymptotic entropy}

The purpose of this section is to review the notion of entropy and to prove Proposition 3.3 below, which gives a condition for upper semicontinuity of the asymptotic entropy of a random walk on a group.

Through the rest of the section we assume $\left\{\mu_{i}\right\}, \mu, v$ to be non-negative measures supported on a countable set. Recall that the entropy of a finite non-negative measure $\mu$ supported on a countable set $G$ is defined by

$$
H(\mu)=\sum_{x \in G}-\mu(x) \log \mu(x),
$$

where by convention $0 \log 0=0$. Throughout this paper all measures to which we apply our results will have finite entropy, though the general statements below make sense and are correct even if we allow measures with infinite entropy. The entropy $H(X)$ of a discrete random variable $X$ is given by the entropy of its distribution; the entropy $H\left(X_{1}, \ldots, X_{n}\right)$ of more random variables is given by the entropy of the joint distribution of the $X_{i}$. In order to define conditional entropy for two random variables $X, Y$, let $f(y)$ denote the entropy of the conditional distribution of $X$ given $Y=y$. Then the conditional entropy of $X$ given $Y$ is defined as $H(X \mid Y):=\mathbb{E} f(Y)$.

The conditional entropy satisfies

$$
H(X, Y)=H(X \mid Y)+H(Y) .
$$

A useful and easy fact is that among measures supported on a given finite set, the one having maximal entropy is the uniform measure on that set. Another well known fact, 
which is relevant to our cause is that among all measures supported on the non-negative integers with given expectation $M$, the entropy is maximized by the geometric distribution with $P(\mu=k)=\frac{1}{1+M}\left(\frac{M}{M+1}\right)^{k}(k=0,1,2, \ldots)$. In particular, this gives the following fact, which be of use later on:

Lemma 3.1. For any random variable $\tau$ supported on $\mathbb{N}$ we have

$$
H(\tau) \leq 2 \log (\mathbb{E} \tau+2)
$$

Define the asymptotic entropy of a sequence of random variables $X_{n}$ as

$$
\limsup _{n \rightarrow \infty} \frac{1}{n} H\left(X_{n}\right)
$$

If a random walk on a group $G$ has i.i.d. steps with distribution given by $\mu$, its asymptotic entropy is given by

$$
H_{\infty}(\mu)=\lim _{n \rightarrow \infty} \frac{1}{n} H\left(\mu^{* n}\right),
$$

where $\mu^{* n}$ is the $n$-fold convolution of $\mu$, or equivalently, the distribution of the $n$th step of the random walk. The limit exists by subadditivity (it is easy to show that $H\left(X_{n+m}\right) \leq$ $\left.H\left(X_{n}\right)+H\left(X_{m}\right)\right)$. In particular, if $H(\mu)<\infty$ then $H_{\infty}(\mu)$ is also finite.

Recall that a set $\left\{\mu_{i}\right\}$ of probability measures is tight if for every $\varepsilon$ there exists a finite set $K$ so that $\sum_{x \notin K} \mu_{i}(x)<\varepsilon$ for all $i$.

We say that a set $\left\{\mu_{i}\right\}$ of probability measures is entropy-tight if for every $\varepsilon$ there exists a finite set $K$ so that for all $i$,

$$
\sum_{x \notin K}-\mu_{i}(x) \log \mu_{i}(x)<\varepsilon
$$

In other words, entropy-tightness means the uniform integrability of the function $\mu_{i}(x) \log \mu_{i}(x)$ with respect to counting measure. The importance of entropy-tightness comes from the following direct application of Vitali's convergence lemma.

Lemma 3.2. Assume that $\mu_{n} \rightarrow \mu$ weakly. Then $H\left(\mu_{n}\right) \rightarrow H(\mu)$ if and only if the sequence $\mu_{n}$ is entropy-tight.

The aim of this section is to prove the following result:

Proposition 3.3. If $\mu_{i} \rightarrow \mu$ and $\left\{\mu_{i}\right\}$ is entropy-tight, then $\lim \sup H_{\infty}\left(\mu_{i}\right) \leq H_{\infty}(\mu)$.

We begin with two lemmas.

Lemma 3.4. For any two measures $\mu$ and $v$ we have $H(\mu * v) \leq|v| H(\mu)+|\mu| H(v)$. 
Proof. For the product measure $\mu \times v$ we have

$$
\begin{aligned}
H(\mu \times v) & =\sum_{x, y} \mu(x) v(y) \log (\mu(x) v(y)) \\
& =\sum_{x, y} \mu(x) v(y) \log \mu(x)+\sum_{x, y} \mu(x) v(y) \log v(y)=|\mu| H(v)+|v| H(\mu) .
\end{aligned}
$$

Now by subadditivity of the function $-x \log x$ we have $H(\mu * \nu) \leq H(\mu \times \nu)$. The lemma follows.

Lemma 3.5. If a family $\left\{\mu_{i}\right\}$ of probability measures is both tight and entropy-tight then so is the set $\left\{\mu_{i_{1}} * \cdots * \mu_{i_{n}}\right\}$ of all their $n$-fold convolutions.

Proof. It suffices to prove this for $n=2$, with larger $n$ following by induction. For a measure $\mu$ we denote the measure restricted to $A$, without normalization, by $\mu^{A}$.

Fix some $\varepsilon>0$. The conditions imply that there is a finite set $K$ such that for all $i$,

$$
\left|\mu_{i}^{K^{c}}\right|=\mu_{i}\left(K^{c}\right)<\varepsilon, \quad H\left(\mu_{i}^{K^{c}}\right)<\varepsilon .
$$

For any two measures $\mu, v$ from the set we have

$$
\mu * v=\mu^{K} * v^{K}+\mu^{K^{c}} * v^{K}+\mu^{K} * v^{K^{c}}+\mu^{K^{c}} * v^{K^{c}} .
$$

Consider the finite set $B=K * K$, and note that the support of $\mu^{K} * v^{K}$ is contained in $B$. It follows that

$$
\left|(\mu * v)^{B^{c}}\right| \leq\left|\mu^{K^{c}} * v^{K}+\mu^{K} * v^{K^{c}}+\mu^{K^{c}} * v^{K^{c}}\right|<2 \varepsilon+\varepsilon^{2},
$$

which can be made arbitrarily small, hence the convolutions form a tight family.

Note that for $a, b \geq 0$ we have $-a \log a-b \log b>-(a+b) \log (a+b)$, so that entropy of measures is sub-additive. Note also that entropy-tightness implies that for some $M$, we have $H\left(\mu_{i}\right) \leq M$ for every $i$.

It follows using Lemma 3.4 that

$$
H\left((\mu * v)^{B^{c}}\right) \leq H\left(\mu^{K^{c}} * v^{K}+\mu^{K} * v^{K^{c}}+\mu^{K^{c}} * v^{K^{c}}\right)<2 M \varepsilon+2 \varepsilon+2 \varepsilon^{2} .
$$

Since this too can be made arbitrarily small, the convolutions are also entropy-tight.

Proof of Proposition 3.3. Fix some $n$. By Lemma 3.5 the set $\left\{\mu_{i}^{* n}\right\}_{i}$ is entropy-tight, and so by Lemma 3.2 we have

$$
H\left(\mu^{* n}\right)=\lim _{i \rightarrow \infty} H\left(\mu_{i}^{* n}\right) .
$$

The sequence $H\left(\mu_{i}^{* n}\right)$ is subadditive, and therefore

$$
H_{\infty}\left(\mu_{i}\right) \leq \frac{1}{n} H\left(\mu_{i}^{* n}\right) .
$$

Taking the lim sup of both sides we get

$$
\limsup _{i \rightarrow \infty} H_{\infty}\left(\mu_{i}\right) \leq \frac{1}{n} \limsup _{i \rightarrow \infty} H\left(\mu_{i}^{* n}\right)=\frac{1}{n} H\left(\mu^{* n}\right) .
$$

Since $n$ is arbitrary, we can take a limit as $n \rightarrow \infty$ to conclude the proof. 


\section{Random walks and the ascension operator}

In our proof we use the method, introduced by Bartholdi and Virág (2005), of studying a random walk on an automaton group by looking at its induced action on the subtree above a vertex $v$, specifically at times $n$ at which $X_{n}$ fixes $v$.

Random walks on quotients and subgroups. Let $\left\{X_{n}\right\}_{n \geq 0}$ be a random walk on a countable group $G$ started at $X_{0}=1$.

If $N$ is a normal subgroup of $G$, and $K=G / N$, then $X$ has a canonical projection to $K$, namely the cosets $Y_{n}=N X_{n}$. We call this the quotient random walk on $G / N$.

A set $S \subset G$ is called recurrent (for a random walk $X$ ) if $X$ visits $S$ infinitely often with probability one. For example, every finite index subgroup is recurrent in $G$. For a recurrent subgroup $S$ the steps at which the random walk visits $S$ form a random walk $Y$ on $S$ (i.e. if $\tau_{n}$ is the $n$th visit to $S$, let $Y_{n}=X_{\tau_{n}}$ ). We call this the induced random walk on $S$.

The ascension operator. Given an automaton group $A$ and a vertex $j \in \mathbb{T}_{m}$, consider the stabilizer subgroup $A_{j}$ of $j$. Consider also the subgroup $A_{j}^{\prime}$ which is the stabilizer of the entire subtree above vertex $j$. Then $A_{j}^{\prime}$ is normal in $A_{j}$. For $g \in A_{j}$ the coset $g A_{j}^{\prime}$ consists of all elements with the same action $g(j)$ on the subtree above $j$. Since $A$ is an automaton group, $g(j) \in A$, hence the group $A_{j} / A_{j}^{\prime}$ is canonically isomorphic to a subgroup of $A$.

We now specialize to vertex $j=0$ in the first level of $\mathbb{T}_{m}$. Given a random walk on $A$ with step distribution $\mu$, we can consider the induced walk on $A_{0}$, and then its quotient walk on $A_{0} / A_{0}^{\prime}$. By the above, this again can be viewed as a random walk on $A$ with step distribution $\mu^{\prime}$. The ascension operator $T$ is the operator that maps each probability measure $\mu$ on $A$, to the measure $\mu^{\prime}$ above. If $\tau_{n}$ are the times at which $X_{\tau_{n}}$ fixes vertex 0 , then the actions of $X_{\tau_{n}}$ on the subtree above 0 are a random walk with step distribution $T \mu$.

We say that a measure $\mu$ is transitive on level $k$ if the group generated by its support acts transitively on that level of $\mathbb{T}_{m}$. We will use the following entropy inequality.

Theorem 4.1 (Kaimanovich (2005, Theorem 3.1)). Assume that a probability measure $\mu$ is transitive on the first level. Then the asymptotic entropies satisfy $H_{\infty}(\mu) \leq H_{\infty}(T \mu)$.

The upper semicontinuity of asymptotic entropy (Proposition 4) yields the following.

Theorem 4.2 (Asymptotic entropy of automaton groups). If the group generated by the support of $\mu$ is transitive on all levels and the sequence $\left\{T^{k} \mu\right\}$ is entropy-tight, then for any subsequential limit point $v$ we have

$$
H_{\infty}(\mu) \leq H_{\infty}(v)
$$

Proof. The transitivity of $\mu$ implies that $T^{k} \mu$ is transitive on the first level for all $k$. Repeated application of Theorem 4.1 shows that for each $k$,

$$
H_{\infty}(\mu) \leq H_{\infty}\left(T^{k} \mu\right)
$$

Taking lim sup along the subsequence converging to $v$ and using Proposition 3.3 gives

$$
H_{\infty}(\mu) \leq \lim \sup H_{\infty}\left(T^{k} \mu\right) \leq H_{\infty}(v) .
$$




\section{Mother groups}

The $(m, r)$-mother group, denoted $\mathfrak{M}_{m, r}$, is defined as the group generated by the automaton with the following states:

$$
\begin{aligned}
\alpha_{k, \sigma} & =\left\langle\left\langle\alpha_{k, \sigma}, \alpha_{k-1, \sigma}, 1, \ldots, 1\right\rangle\right\rangle, \quad 0 \leq k \leq r, \\
\alpha_{-1, \sigma} & =\sigma, \\
\beta_{k, \rho} & =\left\langle\left\langle\beta_{k, \rho}, \beta_{k-1, \rho}, 1, \ldots, 1\right\rangle\right\rangle, \quad 1 \leq k \leq r, \\
\beta_{0, \rho} & =\left\langle\left\langle\beta_{0, \rho}, 1, \ldots, 1\right\rangle\right\rangle,
\end{aligned}
$$

where $\sigma, \rho \in \operatorname{Sym}(m)$ are arbitrary, subject to $0 . \rho=0$. The number of states in $\mathfrak{M}_{m, r}$ as defined here is $m !(r+2)+(m-1) !(r+1)$. The same group can be generated by a smaller set of elements by taking $\sigma, \rho$ only in a minimal, 2 -element set of generators of $\operatorname{Sym}(m)$ and $\operatorname{stab}(0) \subset \operatorname{Sym}(m)$ respectively. This would give a generating set of size $4 r+6$. However, our original choice will prove more suited to our purpose.

The actions of $\alpha_{k, \sigma}$ and $\beta_{k, \rho}$ on a word have simple descriptions. Both read the word and make no changes up to the $(k+1)$ th non-zero letter.

- If the first $k+1$ non-zero letters in a word are all 1 , then $\alpha_{k, \sigma}$ permutes the next letter by $\sigma$. Otherwise it does nothing.

- If the first $k$ non-zero letters in a word are all 1 , then $\beta_{k, \rho}$ permutes the next non-zero letter by $\rho$. Otherwise it does nothing.

Thus both affect only the $(k+1)$ th non-zero letter and the letter immediately following it.

Note that $\alpha_{k, \sigma}$ and $\beta_{k, \rho}$ both have self-loops and are of degree $k$, so they have hierarchy level $\tilde{k}$.

Theorem 5.1 (Mother groups contain all). Every degree-r automaton group is isomorphic to a subgroup of $\mathfrak{M}_{m, r}$ for some $m$.

Note that $m$ is generally not the same as the degree of the tree on which the automaton acts.

Proof. We prove that there exist $m^{\prime}, m^{\prime \prime}$ so that each of the following groups can be isomorphically embedded in the next: $A \subset G_{m^{\prime}, r} \subset \mathfrak{M}_{m^{\prime}, r}^{*} \subset \mathfrak{M}_{m^{\prime \prime}, r}$. The intermediate groups are defined below. The three containments are proved in Lemmas 5.2, 5.6, 5.7.

\section{The first reduction}

For each hierarchy level $h \in\{-\tilde{1},-1, \tilde{0}, 0, \tilde{1}, 1, \tilde{2}, 2, \ldots\}$ we define an automaton group as follows: $G_{m,-\tilde{1}}=\{1\}$, and $G_{m,-1}=\operatorname{Sym}(m)$. For any $r \geq 0$ the group $G_{m, \tilde{r}}$ is obtained by adding to $G_{m, r-1}$ all possible elements of the form

$$
g=\left\langle\left\langle g_{0}, \ldots, g_{m-1}\right\rangle\right\rangle \sigma
$$

(with $\sigma \in \operatorname{Sym}(m)$ ) satisfying the following: there is a unique $i$ such that $g_{i}=g$ and $g_{j} \in G_{m, r-1}$ for $j \neq i$. 
Similarly, for $r \geq 0$ we define $G_{m, r}$ by adding to $G_{m, \tilde{r}}$ all possible elements of the form $g=\left\langle\left\langle g_{0}, \ldots, g_{m-1}\right\rangle \sigma\right.$ where $\sigma \in \operatorname{Sym}(m)$ and $g_{j} \in G_{m, \tilde{r}}$ for all $j$.

Since they are larger than the mother groups, and are also predecessors of the mother groups, in this section we will refer to the groups $G_{m, h}$ as grandmother groups.

Lemma 5.2. Let $A$ be an automaton with all states having hierarchy level at most $h$. Then for some $k$, the automaton group corresponding to the $k$-collapse of $A$ is isomorphic to a subgroup of $G_{m^{k}, h}$.

Proof. Let $k \in \mathbb{Z}$ be a multiple of all cycle lengths of $A$ and larger than the length of any simple directed path in $A$. The $k$-collapsed version $A^{\prime}$ of $A$ has the property that all of its cycles are loops. States with loops have a single loop only, since otherwise $A$ would be exponential.

A state of a given hierarchy level can only point to a state from a lower level, except for states with loops that also point to themselves. By construction, the grandmother group $G_{m^{\prime}, r}$ contains all possible elements of this form.

\section{Extended mother groups}

Let $W_{k}$ denote the finite subgroup of $\operatorname{Aut}\left(\mathbb{T}_{m}\right)$ for which all sections at level $k$ are the identity.

Definition 5.3. The extended mother groups $\mathfrak{M}_{m, \tilde{r}}^{*}, \mathfrak{M}_{m, r}^{*}$ are defined the same way as the ordinary mother groups $\mathfrak{M}_{m, r}$, except that in the definition of the $\alpha_{\sigma, \ell}$ states (3), $\sigma$ ranges over all elements of $W_{r+2}\left(\right.$ for $\mathfrak{M}_{m, \tilde{r}}^{*}$ ) and $W_{r+3}$ (for $\mathfrak{M}_{m, r}^{*}$ ). (The definition of the $\beta_{\rho, \ell}$ states remains unchanged.)

The extended mother groups are nested:

$$
\mathfrak{M}_{m, k-1}^{*} \subset \mathfrak{M}_{m, \tilde{k}}^{*} \subset \mathfrak{M}_{m, k}^{*}
$$

Moreover, we have the following lemma.

Lemma 5.4. If $g_{0}, \ldots, g_{m-1} \in \mathfrak{M}_{m, \tilde{r}}^{*}$, then $g=\left\langle\left\langle g_{0}, \ldots, g_{m-1}\right\rangle\right\rangle \in \mathfrak{M}_{m, r}^{*}$.

Proof. By taking products, it suffices to prove this for the case when all $g_{i}$ are the identity except for one, which is a state of $\mathfrak{M}_{m, \tilde{r}}^{*}$. Moreover, since $\operatorname{Sym}(m) \subset \mathfrak{M}_{m, r}^{*}$, by conjugating by a transposition $\tau \in \operatorname{Sym}(m)$, we may assume that the non-identity entry is $g_{0}$.

We now prove the claim by induction on the degree of $g_{0}$. Consider first elements of type $\alpha$. For $g_{0}=\alpha_{-1, \sigma}=\sigma$ this holds by the definition of $\mathfrak{M}_{m, r}^{*}$ (this is the reason for the choice of $W_{r+3}$ in the definition). For higher degree states, by definition

$$
\alpha_{k, \sigma}=\left\langle\left\langle\alpha_{k, \sigma}, \alpha_{k-1, \sigma}, 1, \ldots, 1\right\rangle\right\rangle
$$

so

$$
\left\langle\left\langle\alpha_{k, \sigma}, 1, \ldots, 1\right\rangle\right\rangle=\alpha_{k, \sigma}\left\langle\left\langle 1, \alpha_{k-1, \sigma}, 1, \ldots, 1\right\rangle\right\rangle^{-1} .
$$

Now, $\alpha_{k, \sigma} \in \mathfrak{M}_{m, \tilde{r}}^{*} \subset \mathfrak{M}_{m, r}^{*}$, and the induction hypothesis implies $\left\langle\left\langle 1, \alpha_{k-1, \sigma}, 1, \ldots, 1\right\rangle\right.$ $\in \mathfrak{M}_{m, r}^{*}$ as well, hence so is their product.

The proof for type $\beta$ states is trivial since their definition remains the same as in the original mother groups. 
Lemma 5.5. If $g_{1}, \ldots, g_{m} \in \mathfrak{M}_{m, r-1}^{*}$, and $\rho \in \operatorname{Sym}(m)$ has $0 . \rho=0$, then

$$
a=\left\langle\left\langle a, g_{1}, \ldots, g_{m}\right\rangle \rho \in \mathfrak{M}_{m, \tilde{r}}^{*} .\right.
$$

Proof. If $g$ is a generator of $\mathfrak{M}_{m, r-1}^{*}$ then by definition $a=\left\langle\langle a, g, 1, \ldots, 1\rangle \in \mathfrak{M}_{m, \tilde{r}}^{*}\right.$. Multiplying such generators shows that this is true for any $g \in \mathfrak{M}_{m, r-1}^{*}$. Define $a_{i}=$ $\left\langle a_{i}, g_{i}, 1, \ldots\right\rangle$. We have

$$
a=\left(\prod_{i} a_{i}^{(1 i)}\right) \rho
$$

where $(1 j)$ is the transposition. Since $\beta_{0,(1 j)}$ and $\beta_{0, \rho}$ are in $\mathfrak{M}_{m, \tilde{r}}^{*}$, it follows that $a \in$ $\mathfrak{M}_{m, \tilde{r}}^{*}$ as claimed.

The key step in the following construction is a conjugation due to Bartholdi et al. (2008), where it was used for bounded automaton groups. Consider the automorphism

$$
\delta=\left\langle\left\langle\delta, \delta \gamma^{-1}, \delta \gamma^{-2}, \ldots\right\rangle\right\rangle \sigma
$$

where $\gamma$ is the cyclic shift $(012 \ldots m-1)$.

Lemma 5.6. For every hierarchy level $h$ the conjugated grandmother group $G_{m, h}^{\delta}$ is a subgroup of the mother group $\mathfrak{M}_{m, h}^{*}$.

Proof. The proof proceeds by induction on $h$. On each level, it suffices to show this for the group generators. For hierarchy level -1 we see that

$$
\sigma^{\delta}=\left\langle\left\langle\gamma^{0-0 . \sigma}, \ldots, \gamma^{m-1-(m-1) . \sigma}\right\rangle \sigma\right.
$$

is an element of $W_{2}=\mathfrak{M}_{m,-1}^{*}$.

Assume the assertion holds for hierarchy level $r-1$. Let $g=\left\langle\left\langle g_{0}, \ldots, g_{m-1}\right\rangle \sigma\right.$ be a generator of $G_{m, \tilde{r}}$. Assume that $g_{k}=g$ is the loop at element $g$. We need to prove that $g^{\delta}$ is an element of $\mathfrak{M}_{m, \tilde{r}}^{*}$, for which it suffices to show that this holds for $h=\gamma^{k} g^{\delta} \gamma^{-k . \sigma}$. We have

$$
h=\left\langle\left\langle h_{0}, \ldots, h_{m-1}\right\rangle \gamma^{k} \sigma \gamma^{-k . \sigma}, \quad \text { where } \quad h_{i}=\gamma^{i+k} g_{i+k}^{\delta} \gamma^{-(i+k) . \sigma} .\right.
$$

Now $h_{0}=h$, the $h_{1}, h_{2}, \ldots$ are in $\mathfrak{M}_{m, r-1}^{*}$, and $\gamma^{k} \sigma \gamma^{-k . \sigma}$ fixes 0 . Thus by Lemma 5.5 we deduce that $h \in \mathfrak{M}_{m, \tilde{r}}^{*}$.

To get from hierarchy level $\tilde{r}$ to hierarchy level $r$, suppose that $g=\left\langle\left\langle g_{0}, \ldots, g_{m-1}\right\rangle\right\rangle \sigma$ where $g_{i}$ are generators of $\mathfrak{M}_{m, \tilde{r}}^{*}$. Then

$$
g^{\delta}=\left\langle\left\langle g_{0}^{\delta \gamma^{0}}, \ldots, g_{m-1}^{\delta \gamma^{m-1}}\right\rangle \sigma^{\delta} \in \mathfrak{M}_{m, r}^{*}\right.
$$

by the inductive hypothesis and Lemma 5.4. 
Embedding in $\mathfrak{M}$

The extended mother groups contain the ordinary mother groups, but there are also embeddings in the other direction, as the following lemma shows.

Lemma 5.7. The $r+3$-collapsed version of $\mathfrak{M}_{m, r}^{*}$ is a subgroup of $\mathfrak{M}_{m^{r+3}, r}$.

Proof. It suffices to show the inclusion of $r+3$-collapsed generators. Let $m^{\prime}=m^{r+3}$. Clearly, the $r+3$-collapse of any element in $W_{r+3}$ is just an element in $\operatorname{Sym}\left(m^{\prime}\right)$. The $r+3$-collapsed version of any other state of type $\alpha$ is of the form

$$
a=\left\langle\left\langle a, g_{1}, \ldots, g_{m^{\prime}-1}\right\rangle \sigma\right.
$$

where $\sigma \in \operatorname{Sym}\left(m^{\prime}\right)$ and the $g_{i}$ 's are states of lower degree than $a$. Just as in Lemma 5.5, we define $a_{i}$ from $g_{i}$ by

$$
a_{i}=\left\langle\left\langle a_{i}, g_{i}, 1, \ldots, 1\right\rangle\right.
$$

and we note that $a_{i}$ is a state of $\mathfrak{M}_{m^{\prime}, r}$. Further $\left\langle\langle 1, \ldots, 1\rangle \sigma\right.$ is just $\alpha_{-1, \sigma}$ and so is also in $\mathfrak{M}_{m^{\prime}, r}$. Thus we can conjugate by the transposition (1i) and write

$$
a=\left(\prod_{i=1}^{m-1} a_{i}^{(1 i)}\right) \sigma
$$

as an element of $\mathfrak{M}_{m^{\prime}, r}$. The proof for states of type $\beta$ uses similar arguments.

\section{Level subgroups}

Observe that the group of automorphisms of the first two levels of $\mathbb{T}_{m}$ fixing 0 and its children is isomorphic to $\operatorname{Sym}(m) 2 \operatorname{Sym}(m-1)$. (We will interpret elements in $\operatorname{Sym}(m-1)$ as acting on $\{1, \ldots, m-1\}$.)

For each $\sigma \in \operatorname{Sym}(m)$ ? $\operatorname{Sym}(m-1)$ and each word $w$ in the symbols $\{1, \ldots, m-1\}$ let $\lambda_{w, \sigma}$ denote the element of $\operatorname{Aut}\left(\mathbb{T}_{m}\right)$ acting as follows: If the first $|w|$ non-zero letters agree with $w$, then $\lambda_{w, \sigma}$ permutes the $(|w|+1)$ th non-zero letter and the following letter by $\sigma$. Otherwise $\lambda_{w, \sigma}$ does nothing. (E.g. $\lambda_{21,(01)(12)}(\cdots 001020010)=\cdots 012020010$ and $\lambda_{21,(01)(12)}(\cdots 002010010)=\cdots 002010010$. $)$

For a word $w$ of length $k$, define the group $\mathfrak{L}_{m, k}^{w}$ generated by $\lambda_{w, \sigma}$ as $\sigma$ ranges over $\operatorname{Sym}(m) \gtrless \operatorname{Sym}(m-1)$. Define $\mathfrak{L}_{m, k}$ to be the group generated by the $\mathfrak{L}_{m, k}^{w}$ for all words $w$ of length $k$. Define further $\mathfrak{L}_{m,-1}=\operatorname{Sym}(m)$.

Later, we will consider random walks on the mother group whose step distribution is a convex combination of uniform measures on the subgroups $\mathfrak{L}_{m, k}$ for various $k$ 's.

Lemma 5.8. For each $w, \mathfrak{L}_{m, k}^{w} \approx \operatorname{Sym}(m)$ i $\operatorname{Sym}(m-1)$. The group $\mathfrak{L}_{m, k}$ is a subgroup of $\mathfrak{M}_{m, k}$ and is the direct product of $\mathfrak{L}_{m, k}^{w}$ for $w \in\{1, \ldots, m-1\}^{k}$. Moreover, the mother group $\mathfrak{M}_{m, k}$ is generated by the subgroups $\left\{\mathfrak{L}_{m, \ell}\right\}_{\ell \leq k}$. 
Proof. The structure of $\mathfrak{L}_{m, k}^{w}$ follows from $\lambda_{w, \sigma} \lambda_{w, \sigma^{\prime}}=\lambda_{w, \sigma \sigma^{\prime}}$. Generators corresponding to different words $w$ of equal length commute, hence $\mathfrak{L}_{m, k}$ is the direct product of the $\mathfrak{L}_{m, k}^{w}$ 's.

For $w=11 \ldots 1$ of length $k$ we see that $\mathfrak{L}_{m, k}^{w}$ is generated by the $\beta_{k, \rho}$ and $\alpha_{k, \sigma}$ 's, hence it is a subgroup of $\mathfrak{M}_{m, k}$.

Now let $w$ be a general word of length $k$, and note that the automorphism

$$
b=\beta_{k-1,\left(1 w_{k}\right)} \cdots \beta_{0,\left(1 w_{1}\right)}
$$

changes only the first $k$ non-zero letters, and if they are all 1 it changes them to the letters of $w$. Thus $\mathfrak{L}_{m, k}^{w}$ is the conjugate by $b$ of $\mathfrak{L}_{m, k}^{11 \ldots 1}$, and therefore $\mathfrak{L}_{m, k}^{w} \subset \mathfrak{M}_{m, k}$ for any $w$.

Note that all elements in $\mathfrak{L}_{m, \ell}$ have degree at most $\ell$. Also since for any $\sigma, \rho \in$ $\operatorname{Sym}(m)$ with $\rho .0=0$ the generators $\alpha_{\ell, \sigma}, \beta_{\ell, \rho}$ are in $\mathfrak{L}_{m, \ell}$, the mother group $\mathfrak{M}_{m, k}$ is generated by the subgroups $\left\{\mathfrak{L}_{m, l}\right\}_{\ell \leq k}$.

From now on, we will use the shorthand notation $\mathfrak{L}_{k}$ and $\mathfrak{M}_{k}$ for $\mathfrak{L}_{m, k}$ and $\mathfrak{M}_{m, k}$ respectively.

\section{Patterns}

The random walks on the mother groups that we consider below have a step distribution that is a mixture of the uniform measures $\overline{q_{i}}$ on the finite level subgroups $\mathfrak{L}_{i}$, and convolutions of these measures. It is convenient to think of the measures $\overline{q_{i}}$ as elements of the group algebra $\mathbb{R} \mathfrak{M}_{k}$. Note that every element of $g \in \mathfrak{L}_{i}$ for $i \geq 0$ is of the form $g=\left\langle\left\langle g, g_{1}, \ldots, g_{m}\right\rangle\right\rangle$ with $g_{j} \in \mathfrak{L}_{i-1}$. Choosing an element of $\mathfrak{L}_{i}$ uniformly corresponds to choosing the $g_{j}$ 's uniformly from $\mathfrak{L}_{i-1}$. This property is central to the control of the ascension of measures that we study.

The elements $\overline{q_{i}} \in \mathbb{R} \mathfrak{M}_{k}$ satisfy the relations ${\overline{q_{i}}}^{2}=\overline{q_{i}}$ (being uniform measures on subgroups), and possibly some other relations that are less tractable. Therefore we introduce the more basic semigroup (with identity $\emptyset$ ) $\mathcal{Q}_{k}$ given by the generators $q_{-1}, \ldots, q_{k}$ (which we call pattern letters) and the relations $q_{i}^{2}=q_{i}$. Elements of $\mathcal{Q}_{k}$ are called patterns. We further define $D=D_{k} \subset \mathcal{Q}_{k}$ to be the set of patterns that contain the letter $q_{-1}$.

Since the measures $\overline{q_{i}}$ satisfy all relations satisfied by the $q_{i}$, the map $q_{i} \mapsto \overline{q_{i}}$ extends multiplicatively to a unique semigroup homomorphism $\mathcal{Q}_{k} \rightarrow \mathbb{R} \mathfrak{M}_{k}$. The image $\bar{p}$ of a pattern $p$ is called the evaluation of $p$.

Each pattern is an equivalence class of words in the letters $\left\{q_{-1}, q_{0}, \ldots, q_{k}\right\}$. The equivalence relation is that repetition of a symbol is equivalent to a single instance. For example, for $a, b, c$ in the set of letters, we have abaacaaabba $\equiv$ abacaba. Composition is concatenation. The length of a pattern $p$, i.e. the length of the shortest element of its equivalence class, is denoted $|p|$. The set $\mathcal{Q}_{0}$ is of particular interest to us. It contains patterns in two letters, which must alternate. Thus $\mathcal{Q}_{0}$ has only two patterns of any length. Finally, for a measure $\mu$ on patterns and $\alpha \geq 0$ the $\alpha$-moment of the length is denoted by

$$
\mu\left(|p|^{\alpha}\right)=\sum_{p \in \mathcal{Q}_{k}}|p|^{\alpha} \mu(p) .
$$


The main reason for the definition of patterns is that they behave nicely with respect to the ascension operator. To make this statement more precise, consider a probability measure $\mu$ supported on patterns. It is a convex combination of measures concentrated on a single pattern, and is naturally an element of $\mathbb{R} \mathcal{Q}_{k}$. Its image $\bar{\mu}$ under the quotient map is an element of $\mathbb{R} \mathfrak{M}_{k}$, or more precisely, a probability measure on $\mathfrak{M}_{k}$. The measure $\bar{\mu}$ will also be called the evaluation of $\mu$. It is given by the formula

$$
\bar{\mu}=\sum_{p \in \mathcal{Q}_{k}} \mu(p) \bar{p} \text {. }
$$

Probability measures of the form $\bar{\mu}$ are special. Nevertheless, this class is preserved by the ascension operator $T$. This suggests that the ascension operator can be defined on the level of patterns.

\section{The ascension formula}

Let $\left\{Z_{n}\right\}$ be the random walk on $G$, with steps $X_{n}$, so that $Z_{n}=X_{1} \cdots X_{n}$, and write $X_{n}=\left\langle\left\langle X_{n}(0), \ldots, X_{n}(m-1)\right\rangle \sigma_{n}\right.$. Clearly the trajectory of 0 is itself a Markov chain: if $J_{n}=0 . Z_{n}$ then $J_{n}=J_{n-1} . \sigma_{n}$. The section $Z_{n}(0)$ is not itself a Markov chain (except for very restricted step distributions). However, the pair $\left(J_{n}, Z_{n}(0)\right)$ is a Markov chain, since $Z_{n}(0)=Z_{n-1}(0) X_{n}\left(J_{n-1}\right)$ : to determine the action of $Z_{n}$ on the subtree above 0 we need to know the action of $Z_{n-1}$ on that subtree, and a single section of $X_{n}$, with index given by $0 . Z_{n-1}$.

A key observation used by Kaimanovich (2005) is that if we consider the process $\left\{Z_{n}(0)\right\}$ conditioned on $\left\{J_{n}\right\}$ we get a process with independent increments. Moreover, if $Z_{n}(0)=Y_{1} \cdots Y_{n}$, then $Y_{n}$ depends on $\left\{J_{n}\right\}$ only through the pair $J_{n-1}, J_{n}$. Indeed the law of $Y_{n}$ is the distribution of $Z_{n}\left(J_{n-1}\right)$ conditioned on $J_{n-1} . \sigma_{n}=J_{n}$.

Such a process can be described naturally by the $m \times m$ matrix $M$ with entries

$$
M_{i j}=\mathbb{E} X(i) 1_{\{i . X=j\}}
$$

in the group algebra $\mathbb{R} G$. Here $X \in \mathfrak{M}_{k}$ is a step of the original random walk, and therefore the section $X(i)$ is also in the group. To take its expectation consider $X(i)$ as an element of the group algebra $\mathbb{R} \mathfrak{M}_{k}$. Note that the entries of this matrix are interpreted as subprobability measures. Their total masses $\left\|M_{i j}\right\|_{1}$ are the transition probabilities for the Markov chain $\left\{J_{n}\right\}$. A useful consequence of this definition is that for $\mu=\mu_{1} * \mu_{2}$ we have $M=M_{1} M_{2}$.

For an $m \times m$ matrix $M$, consider its block decomposition

$$
M=\left(\begin{array}{c|c}
M_{00} & M_{0 *} \\
\hline M_{* 0} & M_{* *}
\end{array}\right),
$$

so that $M_{* *}$ is an $(m-1) \times(m-1)$ matrix.

Proposition 6.1. Let $M$ be as above. Then

$$
T \mu=M_{00}+M_{0 *}\left(I-M_{* *}\right)^{-1} M_{* 0},
$$

where $(I-A)^{-1}$ means $I+A+A^{2}+\cdots$. 
This appears as Theorem 2.3 in Kaimanovich (2005). Note that $M_{* *}$ is an $(m-1) \times(m-1)$ matrix with entries in $\mathbb{R} G$. We include the above discussion and the brief proof because similar ideas are used next for measures on patterns.

Proof of Proposition 6.1. With the above notation, consider the trajectory of 0 until the first time $\tau$ such that $J_{\tau}=0$. The probability of a particular trajectory $0=J_{0}, J_{1}, \ldots, J_{\tau}$ $=0$ is the product of the transition probabilities $\left\|M_{i j}\right\|_{1}$ of the $J$ Markov chain. Conditioned on these values, the process $Y_{n}$ is a product of independent samples from the probability measures $M_{i j} /\left\|M_{i j}\right\|_{1}$ corresponding to the transitions. Paths of length $\tau$ correspond to the term $M_{0 *} M_{* *}^{\tau-2} M_{* 0}$, and $\tau=1$ gives the $M_{00}$ term.

\section{Ascension formula for patterns}

Suppose now that $\mu$ is a probability measure on $\mathcal{Q}_{k}$, and consider the random walk on $\mathfrak{M}_{k}$ with step distribution $\bar{\mu}$. This measure has a great deal of symmetry. A uniformly chosen term $X \in \mathfrak{L}_{-1}$ sends any $i$ to a uniform vertex on level 1 of the tree, and has trivial sections $X(i)$. An element $X \in \mathfrak{L}_{\ell}$ for $\ell>0$ stabilizes the first level of the tree, and its sections are $X(0)=X$ and $X(i) \in \mathfrak{L}_{\ell-1}$, again uniformly. Similarly, an element $X \in \mathfrak{L}_{0}$ fixes 0 , permutes $\{1, \ldots, m-1\}$ uniformly, and its sections are $X(0)=X$ and $X(i) \in \mathfrak{L}_{-1}$, again uniformly and independently.

Thus the matrix $M$ defined above is as follows:

- For $\overline{q_{\ell}}, \ell>0$, we get a diagonal matrix, with terms $M_{00}=\overline{q_{\ell}}$ and $M_{i i}=\overline{q_{\ell-1}}$.

- For $\overline{q_{-1}}$ it is the constant matrix $M_{i j}=1 / m$ (entries are the measure of mass $1 / m$ on the identity element of the group).

- For $\overline{q_{0}}$ we have $M_{00}=\overline{q_{-1}}$, and $M_{0 i}=M_{i 0}=0$ for $i \neq 0$. The remaining minor has constant entries: $M_{i j}=\frac{1}{m-1} \overline{q-1}$ for $i, j>0$.

Note that in all cases, all entries $M_{i j}$ are in $\mathbb{R} \overline{\mathcal{Q}_{k}}$.

Next, consider a measure $\bar{p}$ for a pattern $p=q_{\alpha_{1}} \cdots q_{\alpha_{\ell}}$. Since $\bar{p}$ is a convolution of $\overline{q_{\alpha_{i}}}$, the resulting $M$ is a product of the corresponding $M$ 's, and also has entries in $\mathbb{R} \mathcal{Q}_{k}$. Finally, from (4) we see that $M$ is linear in the step distribution. Since $\bar{\mu}$ is a convex combination of $\bar{p}$, we see that $\bar{\mu}$ also gives $M$ with entries in $\mathbb{R} \mathcal{Q}_{k}$.

Further, note that for all $\overline{q_{\ell}}$ the following sets of entries are all constant:

- the first row, except $M_{00}$;

- the first column, except $M_{00}$;

- the main diagonal, except $M_{00}$;

- $M_{i, j}$ for $i \neq j$ and $i, j>0$.

Note that the algebra of $m \times m$ matrices satisfying the bulleted conditions preserves the two-dimensional space of vectors of the form $(x, y, y, \ldots, y)$. Let $R$ be the operator $M$ acting on this space written in the basis $\{(1,0,0, \ldots, 0),(0,1,1, \ldots, 1)\}$. More 
explicitly,

$$
\begin{array}{ll}
R_{00}=M_{00}, & R_{01}=\sum_{j>0} M_{0 j}, \\
R_{10}=M_{10}, & R_{11}=\sum_{j>0} M_{1 j},
\end{array}
$$

where $M$ is defined by (4) for a sample of $\bar{\mu}$. (Taking any $i>0$ in place of 1 will not make a difference.) Matrices with the above properties form an algebra, and therefore the same identities hold for any $\bar{\mu}$.

Since $M \mapsto R$ is a homomorphism of matrix algebras, $\mu \mapsto R$ is a homomorphism from $\mathbb{R} \mathcal{Q}_{k}$ to the matrix algebra of $2 \times 2$ matrices over $\mathbb{R} \mathfrak{M}_{k}$ : if $\mu=\mu_{1} * \mu_{2}$ then $R=R_{1} R_{2}$.

We now introduce the operators $\mathcal{R}_{i j}$ so that for any measure $\mu$ on $\mathcal{Q}_{k}$ we have $R_{i j} \bar{\mu}=$ $\overline{\mathcal{R}_{i j} \mu}$. Since $\mu \mapsto R$ is a homomorphism, it suffices to define $\mathcal{R}$ for patterns of a single letter, and extend it by multiplicativity and linearity to all of $\mathbb{R} \mathcal{Q}_{k}$. It is clear that the following definition satisfies this desire. For $k \geq 0$, define

$$
\mathcal{R}_{i j} q_{k}= \begin{cases}q_{k}, & i=j=0 \\ q_{k-1}, & i=j=1, \\ 0, & i \neq j .\end{cases}
$$

Note that $k=0$ no longer needs special care (an advantage of using $R$ over $M$ ). For $k=-1$ define

$$
\mathcal{R}_{i j} q_{-1}= \begin{cases}\frac{1}{m}, & j=0, \\ \frac{m-1}{m}, & j=1 .\end{cases}
$$

It follows that the total mass of $\mathcal{R}_{i j} v$ for any measure on patterns is given by

$$
\mathcal{R}_{i j} v\left(\mathcal{Q}_{k}\right)=1_{i=j}(1-v(D))+v(D) \frac{(m-1)^{j}}{m},
$$

where $D$ is the set of patterns containing the letter $q_{-1}$. Let $\mathcal{R} \mu$ be the matrix with entries $\mathcal{R}_{i j} \mu$. As noted above, for a pattern $p=q_{\alpha_{1}} \cdots q_{\alpha_{\ell}}$ we have, using matrix multiplication, $\mathcal{R} p=\prod \mathcal{R} q_{\alpha_{i}}$. For measures $\mu$ we define $\mathcal{R} \mu=\sum_{p} \mu(p) \mathcal{R} p$.

A key consequence of this construction is that a version of Proposition 6.1 holds with $\mathcal{R}$. Define the pattern ascension operator by

$$
\mathcal{T} \mu=\mathcal{R}_{00} \mu+\left(\mathcal{R}_{01} \mu\right)\left(1-\mathcal{R}_{11} \mu\right)^{-1}\left(\mathcal{R}_{10} \mu\right),
$$

where $(1-a)^{-1}=1+a+a^{2}+\cdots$.

Proposition 6.2. For a probability measure $\mu \in \mathbb{R} \mathcal{Q}_{k}$ we have $T \bar{\mu}=\overline{\mathcal{T} \mu}$. 
Proof. Let $M$ be the matrix corresponding to $\bar{\mu}$. By Proposition 6.1,

$$
T \bar{\mu}=M_{00}+M_{0 *}\left(I-M_{* *}\right)^{-1} M_{* 0} .
$$

However, since $M$ is in the aforementioned matrix algebra, it is straightforward to verify that $R_{01} R_{11}^{k} R_{10}=M_{0 *} M_{* *}^{k} M_{* 0}$, and hence

$$
T \bar{\mu}=R_{00}+R_{01}\left(I-R_{11}\right)^{-1} R_{10} .
$$

Since $R \bar{\mu}=\overline{\mathcal{R} \mu}$, this implies the proposition.

We now inspect the structure of $\mathcal{R}$ in more detail. Since $\mathcal{R}$ is multiplicative, for some patterns its entries are delta measures:

Lemma 6.3. For a pattern $p=q_{\alpha_{1}} \cdots q_{\alpha_{\ell}}$ that does not contain the letter $q_{-1}$, we have

$$
\mathcal{R}_{i j} p= \begin{cases}p, & i=j=0, \\ q_{\alpha_{1}-1} \cdots q_{\alpha_{\ell}-1}, & i=j=1, \\ 0, & i \neq j .\end{cases}
$$

For a general pattern with decomposition $p=p_{0} q_{-1} p_{1} \cdots q_{-1} p_{\ell}$ into patterns $p_{i} \in D^{c}$ we have

$$
\mathcal{R}_{i j} p=\left(\mathcal{R}_{i i} p_{0}\right) \prod_{i=1}^{\ell-1}\left(\frac{1}{m} \mathcal{R}_{00} p_{i}+\frac{m-1}{m} \mathcal{R}_{11} p_{i}\right)\left(\mathcal{R}_{j j} p_{\ell}\right) .
$$

Proof. Since $\mathcal{R}$ is multiplicative, the first part holds by definition. The second part follows from the first and from $\mathcal{R}_{i j} q_{-1}=(m-1)^{j} / m$.

Define the averaged legacy operator

$$
\mathcal{A}=\sum_{i, j \in\{0,1\}} \frac{(m-1)^{i}}{m} \mathcal{R}_{i j}
$$

so that $\mathcal{A} \mu=\left(\begin{array}{ll}\frac{1}{m} & \frac{m-1}{m}\end{array}\right)(\mathcal{R} \mu)\left(\begin{array}{l}1 \\ 1\end{array}\right)$. Note that when $\mu$ is a probability measure, so are $\sum_{j} \mathcal{R}_{i j} \mu$, and $\mathcal{A} \mu$.

Lemma 6.4. Let $p=p_{0} q_{-1} p_{1} q_{-1} \ldots q_{-1} p_{k}$ where $p_{i} \in D^{c}$ be the decomposition of $p$ into subpatterns not containing $q_{-1}$ (possibly $p_{0}$ or $p_{k}$ are $\emptyset$ ). Then

$$
\mathcal{A} p=\left(\mathcal{A} p_{0}\right) \cdots\left(\mathcal{A} p_{\ell}\right) .
$$

Proof. This follows from the fact that $\mathcal{R}$ is multiplicative. Writing $\mathcal{R} q_{-1}=\left(\begin{array}{l}1 \\ 1\end{array}\right)\left(\begin{array}{ll}\frac{1}{m} & \frac{m-1}{m}\end{array}\right)$ we have

$$
\begin{aligned}
& \prod_{i=0}^{\ell} \mathcal{A} p_{i}=\left(\begin{array}{ll}
\frac{1}{m} & \frac{m-1}{m}
\end{array}\right)\left(\mathcal{R} p_{0}\right)\left(\begin{array}{l}
1 \\
1
\end{array}\right)\left(\begin{array}{ll}
\frac{1}{m} & \frac{m-1}{m}
\end{array}\right)\left(\mathcal{R} p_{1}\right)\left(\begin{array}{l}
1 \\
1
\end{array}\right) \cdots\left(\begin{array}{ll}
\frac{1}{m} & \frac{m-1}{m}
\end{array}\right)\left(\mathcal{R} p_{\ell}\right)\left(\begin{array}{l}
1 \\
1
\end{array}\right)
\end{aligned}
$$

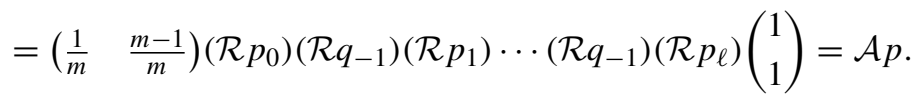




\section{Algorithmic description of $\mathcal{T}$}

We now present an algorithm that uses samples of $\mu$ to get a sample from $\mathcal{T} \mu$. This algorithm is an interpretation of Proposition 6.2, though it is also possible to use it to define $\mathcal{T}$, and derive Proposition 6.2 from it analogously to Proposition 6.1.

To sample $\mathcal{T} \mu$ we consider the Markov chain $\left\{\mathcal{E}_{n}, Q_{n}\right\}_{n>0}$ with state space $\{0,1\} \times \mathcal{Q}_{k}$ and transition probabilities

$$
\mathbb{P}\left(\mathcal{E}_{n}=j, Q_{n}=p \mid \mathcal{E}_{n-1}=i, Q_{n-1}\right)=\left(\mathcal{R}_{i j} \mu\right)(p) .
$$

Lemma 6.5. If $\mathcal{E}_{0}=0$ and $\tau>0$ is minimal such that $\mathcal{E}_{\tau}=0$, then $Q_{1} \cdots Q_{\tau}$ has the law of $\mathcal{T} \mu$.

Proof. This is almost identical to Proposition 6.1. The term $\mathcal{R}_{00} \mu$ corresponds to $\tau=1$, and the term $\left(\mathcal{R}_{01} \mu\right)\left(\mathcal{R}_{11} \mu\right)^{n}\left(\mathcal{R}_{10} \mu\right)$ to $\tau=n+2$.

Since $\mathcal{R} \mu$ is linear, the algorithm above can be broken into the following steps:

- Start with $\mathcal{E}_{0}=0$.

- For $i=1,2, \ldots$ sequentially pick $P_{n} \sim \mu$, and pick $\left(\mathcal{E}_{n}, Q_{n}\right)$ with distribution

$$
\mathbb{P}\left(\mathcal{E}_{n}=j, Q_{n}=p \mid \mathcal{E}_{n-1}=i, Q_{n-1}\right)=\left(\mathcal{R}_{i j} P_{n}\right)(p) .
$$

- At the first time $\tau \geq 1$ so that $\mathcal{E}_{\tau}=0$, stop and return the pattern $Q_{1} \cdots Q_{\tau}$.

\section{Properties of $\mathcal{T}$ and the sequence $\mathcal{T}^{k} \mu$}

In this section we study the evolution of a measure $\mu$ on patterns under iterated ascension.

Proposition 7.1. For a probability measure $\mu$ on patterns, the first moment of the pattern lengths with respect to $\mathcal{T} \mu$ satisfies

$$
\mathcal{T} \mu(|p|) \leq m \mathcal{A} \mu(|p|) .
$$

Proof. Because length is subadditive $(|p q| \leq|p|+|q|)$, (6) implies that the first moment of the length of a sample from $\mathcal{T} \mu$ is bounded above by the first moment of

$$
\theta=\widetilde{\mathcal{R}}_{00} \mu+\widetilde{\mathcal{R}}_{01} \mu\left(1-\widetilde{\mathcal{R}}_{11} \mu\right)^{-1} \widetilde{\mathcal{R}}_{10} \mu,
$$

where $\widetilde{\mathcal{R}}_{i j}$ refer to induced measures on length, and the above formula takes values in the semigroup algebra of $\mathbb{Z}_{+}$. Let $\varphi_{i j}(z)=\sum_{n}\left(\widetilde{\mathcal{R}}_{i j} \mu\right)(n) z^{n}$ denote the generating function for the measure $\widetilde{\mathcal{R}}_{i j} \mu$. The generating function of the measure $\theta$ is

$$
f=\varphi_{00}+\varphi_{01}\left(1-\varphi_{11}\right)^{-1} \varphi_{10} .
$$

The first moment of $\theta$ is given by $f^{\prime}(1)$.

We have formula (5) for the total mass:

$$
\varphi_{i j}(1)=\mathcal{R}_{i j} \mu\left(\mathcal{Q}_{k}\right)=1_{i=j}(1-\mu(D))+\mu(D) \frac{(m-1)^{j}}{m} .
$$

It follows that

$$
f^{\prime}(1)=\varphi_{00}^{\prime}(1)+\varphi_{01}^{\prime}(1)+(m-1)\left(\varphi_{10}^{\prime}(1)+\varphi_{11}^{\prime}(1)\right)=m \mathcal{A} \mu(|p|) .
$$


Lemma 7.2. We have the following (where $D \subset \mathcal{Q}_{k}$ is the set of patterns containing $\left.q_{-1}\right)$ :

(a) For any $x \notin D$ we have $\mathcal{T} \mu(x) \geq \mu(x)$.

(b) If $\mu(D)>0$, then $\mathcal{T} \mu(D)<\mu(D)$.

(c) If $\mu$ is supported on $\mathcal{Q}_{k-1} \cup\left\{q_{k}\right\}$ then so is $\mathcal{T} \mu$, and $\mu\left(q_{k}\right)=\mathcal{T} \mu\left(q_{k}\right)$.

Proof. We refer to the algorithmic description of $\mathcal{T} \mu$ and Lemma 6.3. If the first pattern $P_{1}$ chosen from $\mu$ is in $D^{c}$, then $\mathcal{E}_{1}=0$ and $Q_{1}=P_{1}$ is also the output of the procedure. Claim (a) follows.

For (b), assume that $\mu(p)>0$ for some $p \in D$. Consider $\mathcal{R} p$ as given by Lemma 6.3. Taking only the $(1 / m) \mathcal{R}_{00}$ terms in the product shows that $\mathcal{R}_{00} p$ assigns some positive probability $\delta \geq m^{-\ell}$ to the pattern $p$ with all appearances of $q_{-1}$ deleted. This pattern is in $D^{c}$. Thus $\mathcal{T} \mu\left(D^{c}\right) \geq \mu\left(D^{c}\right)+\delta \mu(p)$.

For (c), note that for such measures, the only way to get $q_{k}$ in the ascension algorithm is if the first pattern selected from $\mu$ is $q_{k}$, in which case it is also the output.

Lemma 7.3. If the measure sequence $\left\{\mathcal{T}^{k} \mu\right\}$ is tight, then its limit exists and is supported on $D^{c}$.

Proof. Since the measures are tight they have subsequential limits that are probability measures. Let $v$ be such a limit. As $\mathcal{T}$ is continuous, there are $k$ so that $v$ and $\mathcal{T}^{k} \mu$ are close enough to have

$$
\mathcal{T}^{k+1} \mu(D)-\mathcal{T} v(D)<\varepsilon .
$$

But again since $v$ is a limit point there is $\ell>k$ so that

$$
v(D)-\mathcal{T}^{\ell} \mu(D)<\varepsilon .
$$

Summing the last two formulae and using that $\mathcal{T}^{\ell} \mu(D) \leq \mathcal{T}^{k+1} \mu(D)$ we get

$$
\nu(D)-\mathcal{T} v(D)<2 \varepsilon .
$$

Since $\varepsilon$ is arbitrary, $\mathcal{T} v(D)=v(D)$. By Lemma 7.2(b), this implies that $v(D)=0$.

Now, $\mathcal{T}^{k} \mu(x)$ is monotone in $k$ for $x \notin D$, so all subsequential limits are equal.

Lemma 7.4. Consider an entropy-tight set $\left\{\mu_{i}\right\}$ of measures on $\mathcal{Q}_{k}$ such that $\mu_{i}(|p|)<M$ for some $M$. Assume further that $H(\bar{p})=o(|p|)$ for all patterns $p \in \bigcup_{i} \operatorname{supp}\left(\mu_{i}\right)$. Then $\left\{\overline{\mu_{i}}\right\}$ is also entropy-tight.

Proof. Denote $K=\left\{g \in \mathfrak{M}_{m, k}:|g| \leq \ell\right\}$, and consider a measure $\mu$ on $\mathcal{Q}_{k}$. By definition,

$$
\bar{\mu}(\cdot)=\sum_{p} \mu(p) \bar{p}(\cdot) .
$$

For compactness, denote $h(x)=-x \log x$, and note that $h$ is subadditive. We have

$$
H_{K^{c}}(\bar{\mu})=\sum_{|g|>\ell} h(\bar{\mu}(g)) \leq \sum_{|g|>\ell} \sum_{p} h(\mu(p) \bar{p}(g)) .
$$


Since $\bar{p}$ is supported on group elements of length at most $|p|$, the above equals

$$
\sum_{|g|>\ell} \sum_{|p|>\ell} h(\mu(p) \bar{p}(g)) \leq \sum_{|p|>\ell} \sum_{g} h(\mu(p) \bar{p}(g)) .
$$

However, since $\bar{p}$ is a probability measure, for any $a>0$ we have

$$
\sum_{g} h(a \bar{p}(g))=-\sum_{g}(\log a+\log \bar{p}(g)) a \bar{p}(g)=h(a)+a H(\bar{p}),
$$

and therefore

$$
H_{K^{c}}(\bar{\mu}) \leq \sum_{|p|>\ell} h(\mu(p))+\sum_{|p|>\ell} \mu(p) H(\bar{p})
$$

Now fix $\varepsilon$. For $\ell$ sufficiently large, the first sum in (9) is uniformly small for all $\mu$ in our entropy-tight family. Also by assumption, if $\ell$ is large enough then either $\mu(p)=0$ or $H(\bar{p})<\varepsilon|p|$. Thus for large enough $\ell$ we have

$$
H_{K^{c}}(\bar{\mu}) \leq \varepsilon+\sum_{|p|>\ell} \mu(p) \varepsilon|p| \leq \varepsilon+M \varepsilon .
$$

Since $\varepsilon$ is arbitrary, this implies that $\left\{\overline{\mu_{i}}\right\}$ is entropy-tight as claimed.

\section{Preliminary results for entropy of patterns}

Here we establish some useful facts about entropy of $\bar{\mu}$ for some measures $\mu$ on patterns. We first recover the following result.

Theorem 8.1 (Bartholdi et al. (2008)). Let $\mu$ be supported on patterns of length 1 in $\mathcal{Q}_{0}$. Then $H_{\infty}(\bar{\mu})=0$, or equivalently $H\left(\bar{\mu}^{* n}\right)=o(n)$.

We present what is essentially the original proof, written in the language of this paper.

Proof. Lemma 7.2(c) shows that $\mathcal{T}^{k} \mu$ is supported on $\left\{q_{0}\right\} \cup \mathcal{Q}_{-1}=\left\{q_{0}, q_{-1}, \varnothing\right\}$. Thus the sequence $\mathcal{T}^{k} \bar{\mu}$ is tight and entropy-tight. Lemma 7.3 shows that the $v=\lim \mathcal{T}^{k} \mu$ exists and is supported on $\left\{q_{0}, \emptyset\right\}$. Thus $\operatorname{supp}(\bar{v})$ is contained in a finite group, and $H_{\infty}(\bar{v})$ $=0$. The result now follows by Theorem 4.2.

Next, we show that evaluations of patterns on $\mathcal{Q}_{0}$ have entropy which is sublinear in their length. We need the following simple lemma.

Lemma 8.2 (Monotonicity of pattern entropy). If $p$ and $r$ are patterns, then

$$
H(\overline{p r}) \geq \max (H(\bar{p}), H(\bar{r})) .
$$

Proof. $\overline{p r}$ is a convex combination of measures of the form $\bar{p} g$ where $g$ is chosen according to $\bar{Q}$. All these measures have the same entropy $H(\bar{r})$. Entropy is a concave function, so by Jensen's inequality

$$
H(\overline{p r}) \geq H(\bar{p}) .
$$

In the same way, $H(\overline{p r}) \geq H(\bar{r})$. 
Lemma 8.3. There is a function $f(n)=o(n)$ such that for any pattern $p \in \mathcal{Q}_{0}$,

$$
H(\bar{p}) \leq f(|p|) .
$$

Proof. Let $\mu$ be the uniform measure on $\left\{q_{-1}, q_{0}\right\}$, and $\mu_{n}=\mu^{* n}$. By concavity of entropy and Jensen's inequality, since $\overline{\mu_{n}}=\sum \mu_{n}(p) \bar{p}$, we have

$$
H\left(\overline{\mu_{n}}\right) \geq \sum_{p \in \mathcal{Q}_{0}} \mu_{n}(p) H(\bar{p}) \geq \sum_{p \in A_{\ell}} \mu_{n}(p) H(\bar{p}),
$$

where $A_{\ell}$ consists of all patterns of length greater than $\ell$. By Lemma 8.2, the entropy of each word of length $n$ is greater than the entropy of each word of length $n-1$ (since patterns in $\mathcal{Q}_{0}$ are just alternating $q_{0}$ 's and $q_{-1}$ 's). It follows that

$$
H\left(\overline{\mu_{n}}\right) \geq \mu_{n}\left(A_{\ell}\right) H\left(\overline{Q_{\ell}^{*}}\right),
$$

where $Q_{\ell}^{*}$ can be either of the two patterns of length $\ell$.

The length of a sample of $\mu_{n}$ is the number of runs in the word $Q_{1} \cdots Q_{n}$ with i.i.d. letters. Since each letter starts a new run with probability $1 / 2$, the length is binomial, and symmetric about $n / 2$. Thus with $n=2 \ell$ we have $\mu_{n}\left(A_{\ell}\right) \geq 1 / 2$. We find

$$
H\left(Q_{\ell}^{*}\right) \leq 2 H\left(\overline{\mu_{2 \ell}}\right)=o(\ell) .
$$

We can also make some conclusions about linear automaton groups. It is not hard to see that the combined supports $\mathfrak{L}_{0}, \mathfrak{L}_{1}$ of the evaluated patterns $\bar{q}_{0}, \bar{q}_{1}$ generate a bounded automaton group. Certain walks on this group also have zero asymptotic entropy.

Lemma 8.4. Any measure $\mu$ on $\mathcal{Q}_{1}$ supported on the patterns $\left\{q_{0}, q_{1}, \emptyset\right\}$ satisfies $H_{\infty}(\bar{\mu})$ $=0$.

Proof. Consider the subgroup of $\mathfrak{M}_{m, 1}$ consisting of all automorphisms of the form

$$
g=\left\langle\left\langle g, g_{1}, \ldots, g_{m-1}\right\rangle \rho,\right.
$$

where 0. $\rho=0$. Note that the support of $\bar{\mu}$ is contained in this subgroup. Consider a random walk $X_{k}$ with step distribution $\bar{\mu}$, and write

$$
X_{k}=\left\langle\left\langle X_{k}, Y_{k}(1), \ldots, Y_{k}(m-1)\right\rangle\right\rangle \rho_{k} .
$$

The key observation is that the distribution of each $Y_{1}(i)$ is $\overline{\mu^{\prime}}$ where $\mu^{\prime}=\mathcal{R}_{11} \mu$. It follows that for each $i>0$ the process $\left(Y_{k}(i), k \geq 0\right)$ is a random walk on $\mathfrak{M}_{m, 0}$ with step distribution $\overline{\mu^{\prime}}$, where $\mu^{\prime}$ is supported on $\left\{q_{-1}, q_{0}, \emptyset\right\}$. By Theorem 8.1, this random walk has zero asymptotic entropy. On the other hand, $X$ is determined by $\rho$ and the $Y_{i}$ 's, so

$$
H\left(X_{k}\right) \leq H\left(\rho_{k}\right)+\sum_{i=1}^{m-1} H\left(Y_{k}(i)\right) \leq \log m !+(m-1) H\left(Y_{k}(1)\right) .
$$

Thus $H_{\infty}(\bar{\mu}) \leq(m-1) H_{\infty}\left(\overline{\mu^{\prime}}\right)=0$, as required. 


\section{The linear-activity case and the main theorem}

The key step in the proof of the main theorem is the following proposition. It is based on the results from the previous sections.

Proposition 9.1. Let $\mu$ be a measure on $\mathcal{Q}_{0} \cup\left\{q_{1}\right\}$ with $\mu(|p|)<\infty$. Then the sequence $\mu_{k}=\mathcal{T}^{k} \mu$ is both tight and entropy-tight.

The tightness of the sequence depends on a certain contracting property of the ascension operator $\mathcal{T}$ for patterns on $\mathcal{Q}_{0}$. Proposition 7.1 gives bounds on moments of $\mathcal{T} \mu$ in terms of moments of $\mathcal{A} \mu$. The next lemma bounds the moments of $\mathcal{A} \mu$.

Lemma 9.2 (Length of legacy). For a probability measure $\mu$ on $\left\{q_{1}\right\} \cup \mathcal{Q}_{0}$, we have

$$
\mathcal{A} \mu(|p|) \leq 1+\frac{m-1}{m^{2}} \mu(|p|)
$$

Proof. The probability measure $\mu$ is a convex combination of delta measures on single patterns. Since $\mathcal{A} \mu$ is linear, and the bounds are all affine in $\mu$, it suffices to prove the claim for delta measures $\mu=p$ supported on a single pattern $p \in\left\{q_{1}\right\} \cup \mathcal{Q}_{0}$.

If $p \in\left\{\emptyset, q_{1}, q_{-1}\right\}$, then $\mathcal{A} \mu$ is supported on patterns of length at most 1 , and the claim is trivial.

Otherwise, $\mu=p$ for some $p \in \mathcal{Q}_{0}$. Let $t>0$ be the number of times $q_{0}$ appears in $p$, so that $|p| \geq 2 t-1$. By (7), a sample of $\mathcal{A} \mu$ is given by $X_{1} \cdots X_{t}$ where $X_{i}$ are i.i.d. with distribution $\mathcal{A} q_{0}=\frac{1}{m} q_{0}+\frac{m-1}{m} q_{-1}$. Each run of $q_{0}$ 's or $q_{-1}$ 's reduces to a single letter, so the length $R$ of $X_{1} \cdots X_{t}$ is given by the number of runs.

Let $N_{i}$ be the indicator of the event that $X_{i}$ starts a new run, so that $R=N_{1}+\cdots+N_{j}$. Then $N_{1} \equiv 1$ and for $1<i \leq t$ we have $\mathbb{E} N_{i}=2(m-1) / m^{2}$. Now,

$$
\mathbb{E} R=1+\frac{2(m-1)}{m^{2}}(t-1) \leq 1+\frac{m-1}{m^{2}}|p| .
$$

Combining Lemma 9.2 with Proposition 7.1 gives the following contraction property of $\mathcal{T}$ :

Corollary 9.3 (Contraction). For any probability measure $\mu$ on $\mathcal{Q}_{0} \cup\left\{q_{1}\right\}$ we have

$$
\mathcal{T} \mu(|p|) \leq \frac{m-1}{m} \mu(|p|)+m .
$$

Proof of Proposition 9.1. Any non-negative sequence satisfying $x_{k+1} \leq c+\alpha x_{k}$ with $\alpha<1$ must be bounded. By Corollary 9.3, the sequence $\mu_{k}(|p|)$ is bounded by some constant $C$, which implies tightness.

For entropy-tightness, since $\mu_{k}$ is supported within $\left\{q_{0}, q_{1}, \varnothing\right\} \cup D$, it suffices to show that the contribution to its entropy from the set $D$ converges to 0 . Let $v_{k}$ denote $\mu_{k}$ conditioned to the set $D$. The contribution to $H\left(\mu_{k}\right)$ from $D$ is given by

$$
\sum_{P \in D}-\mu_{k}(P) \log \mu_{k}(P)=\varepsilon_{k} H\left(v_{k}\right)-\varepsilon_{k} \log \varepsilon_{k} .
$$


We have

$$
v_{k}(|p|) \leq \frac{\mu_{k}(|p|)}{\varepsilon_{k}} \leq \frac{C}{\varepsilon_{k}} .
$$

However, note that $v_{k}$ is supported on patterns of alternating $q_{0}$ 's and $q_{-1}$ 's, so we have

$$
H\left(v_{k}\right) \leq 1+H\left(\tilde{v}_{k}\right),
$$

where $\tilde{v}_{k}$ is the induced measure on lengths. Using Lemma 3.1 we get

$$
H\left(\tilde{v}_{k}\right) \leq 2 \log \left(C / \varepsilon_{k}+2\right) .
$$

Combining this with (10), (11), and Lemma 7.3 which shows that $\varepsilon_{k} \rightarrow 0$, completes the proof.

We are ready to prove the following, which implies Theorem 1.

Theorem 9.4. Let $\mu$ be a probability measure on patterns in $\mathcal{Q}_{0} \cup\left\{q_{1}\right\}$ with $\mu(|p|)<\infty$. The random walk on the linear-activity mother group with step distribution $\bar{\mu}$ has zero asymptotic entropy.

Proof. By Theorem 4.2 it suffices to show that the sequence $T^{k} \bar{\mu}$ is entropy-tight and converges weakly to a measure with zero asymptotic entropy.

Consider first the sequence $\mathcal{T}^{k} \mu$ of measures on patterns. Proposition 9.1 shows this sequence is tight. Lemmas 7.2 and 7.3 imply that $v=\lim \mathcal{T}^{k} \mu$ exists and is supported on $\left\{q_{1}, q_{0}, \emptyset\right\}$. Finally, by Lemma 8.4, evaluations of measures supported on $\left\{q_{1}, q_{0}, \emptyset\right\}$ have zero entropy, so $H_{\infty}(\bar{v})=0$.

It remains to show that $T^{k} \bar{\mu}$ is entropy-tight. This follows from Lemma 7.4, so we verify its conditions. Entropy-tightness and uniformly bounded expected length for $\mathcal{T}^{k} \mu$ are proved in Proposition 9.1. The support of any $\mathcal{T}^{k} \mu$ is contained in $\left\{q_{1}\right\} \cup \mathcal{Q}_{0}$. By Lemma 8.3 the entropy of $p \in\left\{q_{1}\right\} \cup \mathcal{Q}_{0}$ is $o(|p|)$.

Acknowledgments. This research was carried out while all authors were at the University of Toronto. We thank V. Nekrashevych for bringing the example of the long-range group to our attention, as well as an anonymous referee for pointing out a simplification of our proof.

Research of O. Angel was supported in part by NSERC. Research of B. Virág was supported in part by NSF grant.

\section{References}

Adyan, S. I. (1982): Random walks on free periodic groups. Izv. Akad. Nauk SSSR Ser. Mat. 46, 1139-1149 (in Russian) Zbl 0512.60012 MR 0682486

Amir, G., Virág, B. (2011): Positive speed for high-degree automaton groups. arXiv:1102.4979

Bartholdi, L., Grigorchuk, R., Nekrashevych, V. V. (2003): From fractal groups to fractal sets. In: Fractals in Graz 2001, Trends Math., Birkhäuser, Basel, 25-118 Zbl 1037.20040 MR 2091700

Bartholdi, L., Kaimanovich, V. A., Nekrashevych, V. V. (2008): On amenability of automata groups. Duke Math. J. 154, 575-598 Zbl pre05816151 MR 2730578

Bartholdi, L., Virág, B. (2005): Amenability via random walks. Duke Math. J. 130, 39-56 Zbl 1104.43002 MR 2730578 
Benjamini, I., Hoffman, C. (2005): $\omega$-periodic graphs. Electron. J. Combin. 12, Research Paper 46, 12 pp. Zbl 1098.05069 MR 2176522

Glasner, Y., Mozes, S. (2005): Automata and square complexes. Geom. Dedicata 111, 43-64. Zbl 1088.20037 MR 2155175

Grigorchuk, R. I. (1984): Degrees of growth of finitely generated groups and the theory of invariant means. Izv. Akad. Nauk SSSR Ser. Mat. 48, 939-985 (in Russian) Zbl 0583.20023 MR 0764305

Grigorchuk, R., Šunić, Z. (2006): Asymptotic aspects of Schreier graphs and Hanoi towers groups. C. R. Math. Acad. Sci. Paris 342, 545-550 Zbl 1135.20016 MR 2217913

Grigorchuk, R. I., Šunić, Z. (2007): Self-similarity and branching in group theory. In: Groups St. Andrews 2005, London Math. Soc. Lecture Note Ser. 339, Cambridge Univ. Press, 36-95 Zbl 1185.20044 MR 2327317

Grigorchuk, R. I., Żuk, A. (2001): The lamplighter group as a group generated by a 2-state automaton, and its spectrum Geom. Dedicata 87, 209-244 Zbl 0990.60049 MR 1866850

Grigorchuk, R. I., Żuk, A. (2002): On a torsion-free weakly branch group defined by a three state automaton. Int. J. Algebra Comput. 12, 223-246 Zbl 1070.20031 MR 1902367

Guido's book of conjectures (2008): Collected by I. Chatterji, Enseign. Math. (2) 54, 3-189 Zbl 1143.00006

Kaimanovich, V. A. (2005): "Münchhausen trick" and amenability of self-similar groups. Int. J. Algebra Comput. 15, 907-937 Zbl 1168.20308 MR 2197814

Kemp, A. W. (1997): Characterizations of a discrete normal distribution. J. Statist. Plann. Inference 63, 223-229 Zbl 0902.62020 MR 1491581

Mazurov, V. D., Khukhro, E. I. (eds.) (2006): The Kourovka notebook. Unsolved problems in group theory. Russian Acad. Sci., Novosibirsk

Milnor, J. (1968): Growth of finitely generated solvable groups. J. Differential Geom. 2, 447-449 Zbl 0176.29803 MR 0244899

Olshanskii, A. Y. (1980): On the question of the existence of an invariant mean on a group. Uspekhi Mat. Nauk 35, no. 4, 199-200 (in Russian) Zbl 0465.20030 MR 0586204

Ol'shanskii, A. Y., Sapir, M. V. (2002): Non-amenable finitely presented torsion-by-cyclic groups. Publ. Math. Inst. Hautes Études Sci. 96, 43-169 Zbl 1050.20019 MR 1985031

Sidki, S. (2000): Automorphisms of one-rooted trees: growth, circuit structure, and acyclicity. J. Math. Sci. (New York) 100, 1925-1943 Zbl 1069.20504 MR 1774362

Sidki, S. (2004): Personal communication with the last author, at the conference "Geometric Group Theory, Random Walks and Harmonic Analysis", Cortona, June 13-18, 2004

Vorobets, M., Vorobets, Y. (2007): On a free group of transformations defined by an automaton. Geom. Dedicata 124, 237-249 Zbl 1183.20024 MR 2318547 